\section{Barriers to trust}

\section{An outbreak of Ebola highlights the difficulties of implementing public-health measures.}

\section{$\mathrm{T}$} he current outbreak of Ebola virus disease in West Africa, which began last December in Guinea and has since spread to Liberia and Sierra Leone, has already caused 779 cases, including 481 deaths. This makes it much larger than past outbreaks, and it has generated extensive media coverage worldwide.

It is not difficult to explain the macabre interest in Ebola. The virus can kill up to $90 \%$ of the people it infects. It causes a horrible death, with initial symptoms of fever and muscle pain followed by vomiting, diarrhoea, kidney and liver damage, and sometimes profuse internal and external bleeding. Despite these tragic effects, a sense of perspective is required. Since the virus was first discovered in Zaire (now the Democratic Republic of the Congo), there have been only a few dozen sporadic outbreaks - most of them small and largely confined to central Africa.

Ebola is a rare disease that, in some 40 years, has resulted in barely 2,000 deaths. Although even one premature death is too many, the toll of Ebola pales beside the millions killed every year in Africa by other infectious diseases. These include HIV/AIDS, malaria and influenza, as well as measles, diarrhoea and pneumococcal pneumonia, which rarely get such attention from the media. Ebola also poses a very low risk to travellers and those outside the affected region, as long as they take basic precautions against infection.

Ebola seems to hold little or no pandemic threat. Unlike an outbreak of a new pandemic flu strain, which is impossible to contain,

Ebola spreads poorly between people. Its transmission requires direct contact with the bodily fluids of those who are infected, such as saliva, faeces or blood. This also means that, even in the absence of a licensed Ebola vaccine, stopping an outbreak in its tracks should, in principle, be straightforward. The disease can be controlled by public-health measures alone, such as surveillance and diagnosis of those infected and their contacts, prompt quarantine of cases and other basic infec-

"Stopping an Ebola outbreak should, in principle, be straightforward." tion control.

It is not known how the current outbreak began. Fruit bats that serve as a probable reservoir of Ebola are present in the remote forest in southeast Guinea where this outbreak was initially reported, and the disease is thought to have jumped to humans through contact with contaminated bushmeat or infected primates. However, the continuing outbreak, which is now affecting dense urban populations, is mainly being driven by human-to-human spread: traditional burial practices that include contact with corpses, sick people not getting or seeking care, and a lack of basic infection-control measures.

The difficulties in implementing the necessary public-health measures in Guinea, Liberia and Sierra Leone, which are among the poorest countries on the planet, are formidable. Because people in these regions are often poor and many are illiterate, it is not easy to communicate the risk factors for Ebola and the infectioncontrol measures needed - particularly when the disease, and the biohazard suits of health-care workers, can strike terror into the hearts of affected communities. Some workers have reportedly been attacked by locals who believed that they had brought in the disease.

As with social resistance to vaccines in even the richest countries, sometimes diseases continue to cause illness and death - not because of a lack of tools to stop them, but because of the difficulties of conveying public-health measures and overcoming fixed, but misplaced, beliefs.

\section{Be concerned}

\section{A possible link between neonicotinoid pesticide use and a decline in bird numbers is worrying.}

$\mathrm{D}$ oes anything eat wasps? Despite the popularity of the question, which spawned a series of bestselling books, the answer is less interesting than one might think. Yes, lots of things eat wasps - bigger wasps, for a start. Then there is the bee-eater (don't blame it, we chose the name) and hundreds of other bird species. Perhaps one might instead ask, does anything not eat wasps?

Plenty of these birds eat bees, and other insects too. So it is reasonable to ask a different question: if the numbers of these insects fall, will the birds that feed on them have enough to eat?

This is a complicated question, more difficult to answer than it might seem. So the provocative findings published in a paper on Nature's website this week must be taken as a question themselves, rather than a definitive answer. In the paper, researchers suggest that common agricultural pesticides that have long been linked to the decline in some bee species could also be affecting birds (C. A. Hallmann et al. Nature http://dx.doi.org/10.1038/nature13531; 2014).

The suggestion is based on an observed correlation between declines in some farmland bird populations and the use of neonicotinoid pesticides in the Netherlands. The analysis indicates that pesticide use may reduce the amount of prey insects available to birds, causing the observed association, and suggests that neonicotinoids pose an even greater risk to wildlife than previously thought.

Correlation, as every science blogger who reports this story will point out, is not the same as causation. The evidence that the agricultural chemicals can be blamed for the loss of the birds is circumstantial, being based on the finding that the steepest declines are in regions of the country that also show the highest water concentrations of imidacloprid, the most commonly used neonicotinoid. But the authors also rule out confounding effects from other land-use changes or pre-existing trends in bird declines.

Insects form a large part of the diet of many bird species during the breeding season and are important for raising offspring. Nine of the 15 species investigated exclusively eat insects, and all feed insects to their young, which supports the theory that pesticide use is negatively affecting bird populations by depleting their food sources. Still, although many birds do eat bees, none of those studied would ordinarily eat them in any quantity. So a new question must be addressed: if the pesticides are killing the birds, then how are they doing it?

In an accompanying News \& Views article, ecologist Dave Goulson suggests one possibility (D. Goulson Nature http://dx.doi.org/10.1038/ nature 13642; 2014). Almost all of the neonicotinoid used ends up in the soil and water as a pollutant. Some could be taken up by non-crop plants, such as hedgerows. Surrounded by tainted food and water, aquatic insects and grasshoppers, beetles and caterpillars could be poisoned - just as bees are - and so not be around to feed the birds.

The picture is incomplete, and thus Goulson's invocation of Rachel Carson's Silent Spring, a tale of ecological doom, may seem premature. But his is not a lone voice. An international assessment on the likely effect of neonicotinoids, released by scientists (including Goulson) late last month, warned that the consequences for biodiversity and human food supplies could be severe (see go.nature.com/gzhg94). $\rightarrow$ NATURE.COM To comment online, click on Editorials at: go.nature.com/xhunqu
And Europe has already imposed a two-year moratorium on the use of three common neonicotinoids on flowering crops.

So, does anything eat wasps? Yes, and we must do more to ensure that that continues. 